\title{
KINESITHERAPY OF PREMENSTRUAL PAIN IN SPORTS WOMEN
}

\author{
Tatyana Tomova \\ National Sports Academy "Vassil Levski", Sofia, Bulgaria
}

\begin{abstract}
The aim of the present study is to analyze the issue of the syndrome in gynecological practice, particularly for premenstrual pain, and to offer alternative therapy for this condition.

The comparative analysis of the results revealed a positive change in survey indicators, which led to improvement in quality of life as a whole.

The strength of the effect of treatment of Chinese point massage is due to the natural regulation and activation of the hidden reserves of the organism. Its application in practice can only enrich and expand kinesitherapy indications.
\end{abstract}

Key words: Premenstrual pain, Chinese point massage.

\section{INTRODUCTION}

The Premenstrual syndrome (PMS) is a complex and not fully explored problem in the modern medicine. It is among the most common clinical complaints of women's reproductive age.

There are limited studies as it goes to the influence of the symptoms to women's daily activities, but they do confirm that the syndrome leads to interruption in relationships, work and/or social problems (Ford, Lethaby, 2009; Haywood, Slade, 2007; Pinar, Colak, 2011). The monthly expectation of pain affects the woman's overall self-esteem, emotional and mental activity (Goranova, 2006). Due to the insufficiently clarified etiology in traditional medicine, the treatment of PMS is predominantly symptomatic and thoroughly includes medication (analgesics, NSAIDs, hormones, antidepressants, inhibitors, diuretics, vitamins, nutritional supplements, etc.) and in the extremely severe cases, operations. Bearing in mind the potential side effects of these approaches, alternative medicine, massage and physical exercises are increasingly attracting the attention of specialists and women.

In the past few years, Chinese medicine methods have taken its well-deserved place among physical remedies for the prophylaxis and treatment of numerous diseases, especially in pain conditions (Goranova, 2006). The efficacy of acupressure (ACP) has been evaluated in various studies. It is able to reduce the length of the Central Nerve System's response time (Lee et all, 2004). Furthermore, it has been shown that the severity of dysmenorrhea can be reduced rapidly by the application of acupressure. Consequently, this implies a constant search, development and use of various physical means for the adequate treatment of pain syndrome in gynecological practice.

The aim of this study is to investigate the effectiveness of Chinese point massage as it goes to young women with premenstrual pain who are physically active.

\section{METHODOLOGY}

The experiment was conducted in outpatient settings featuring 20 women aged 20 to 30 years old and who suffer from premenstrual pain who are not taking any analgesics. All individuals have signed an informed consent in order to participate in this survey. A Chinese massage - An'mo (Goranova, 1994) - was applied for the treatment of premenstrual pain.

The tasks of the massage included:

1) overcoming the PMS;

2) removing pervasive phenomena;

3) strengthening trophic and exchange processes;

4) activate the hidden reserves in the body;

5) soothing the psyche;

The following indicators were used in order to assess the effectiveness of the applied methodology:

Measuring body circuits: the body circumferences were measured with a centimeter band with an accuracy of $0.5 \mathrm{sm}$. They are as follows: 
- The waist circumference (OT) is measured at the navel level.

- The hip circumference (OX) is measured at the level of the glutaneous fold, etc. breech zone (reflexogenic area of the ovaries).

- Knee circumference (PK) through a patella for a more detailed examination as it is a reflexogenic area of the uterus, and in case of problems, this area deforms significantly and composes the so-called cellulite cushion.

These circuits give an idea to the sites of accumulation to an adipose tissue. They are called cellulite zones (Topuzov, 2000). The risk of a woman having menstrual abnormalities raise by $8 \%$ when the waist is increased by $1 \mathrm{sm}$. With increasing fat content in the woman's body, the risk of menstrual cycle disturbances (MC) enlarges too (Kedicova-Andreeva, 2012).

Assessment of skin fold (ASF) on a 4-point scale in three zones (on both sides of the umbilicus level - from the spina iliaca anterior superior to the ribbed arc, the zone over the trochanter major and the medial part of the patella). The patient is in a baseline bed, engaging the fingers of both hands, a skin fold is gripped and lifted perpendicularly to the body surface, moving up to the test areas. The resistance of the soft tissues to the withdrawal of the skin fold and the severity of the sore sensation is read as follows:

- Grade 0 - no resistance, no pain;

- Grade 1 - with low resistance, with little sense of pain;

- Grade 2 - there is pain and forms the so-called "Orange skin";

- Grade 3 - no skin fold can be gripped, sense of severe pain;

Considering the reflex and segmented structure of the spinal cord nervous system, each pathological site causes reflex changes on the body surface. The discovery of a thicker and more difficult to grip skin fold, indicates a functional disease of the organ which is associated with the reflexive pathway (Kraev, 2016). The tracked skin folds are related to the female reproductive system.

Pain test that is scored on a 4-degree scale: In the study of patients with lower back pain, the individual sensation of pain and sensitivity threshold were also recorded. A subjective assessment was made as it goes to the degree of pain, and it was done by a modification by D. Kostadinov (1978), a classic test of Merl d'Aubine.

- Grade 0 - no pain;

- Grade 1 - weak, dull pain;

- Grade 2 - moderate pain;

- Grade 3 - severe pain

The surveyed indicators were taken at the same time for each of the tested patients. They were evaluated three times during the course of treatment. Initial measurements were performed prior to initiation of treatment, i.e., before the first monitored menstruation (MC). Intermediate measurements were made before the second tracked MC. Final measurements were performed after the last procedure was completed. The CM treatment course started seven days before the supposed date of the forthcoming MC. Procedures were held every day.

The treatment course lasted three months (covering three consecutive $\mathrm{MC}$ ). A late review was performed 1 month after the course of treatment, to keep track of the duration of the effect in result to the applied methodology.

Methodology used: Main Active Points: He'gu LI 4, Guan'yuan CV 4, Qi'hai CV 6, Zu'san'li ST 36, San'yin'jiao SP 6, Tai'xi KI 3, Tai'chong LR 3, Nei'guan PC 6, Zhong' wan CV 12, Tian'shu ST 25, Shan' zhong CV 17 (Goranova, 2006) and also other active points according to accompanying symptoms (Andonova, 2014; Gotova et all, 2015; Gotova, 2015). Therefore, the active points for each woman were individually selected according to the leading symptoms. The combination of the above-mentioned points helps to regulate the main premenstrual symptoms and, most importantly, to control the pain as a leading physical complaint.

Methodological guidelines: The requirements for the application of An'mo massage are based on the method of Goranova (1994), (Goranova, 1994).

\section{Statistical analysis:}

The results obtained are processed with a SPSS 19.0 statistical processing program. The t-criterion of the Student for dependent samples was used. When checking the statistical significance of the results, a guarantee probability $\mathrm{P}>95 \%$ (or significance level $a<0.05)$ was determined. 


\section{RESULTS}

Measuring body circuits: the applied point massage technique did not lead to statistically significant changes in lap reduction. Nevertheless, all three laps decrease smoothly over time and those changes persist for 1 month after the end of the treatment. The circumference of the abdomen through the navel shows a subsequent late therapeutic effect, which is most likely a result from the cumulative effect of the spot massage procedures, which proves that this area responds best. I suppose that reducing the circumference of the abdomen by 1.10 $\mathrm{cm}$ reduces also the risk of developing menstrual disorders (Kedicova-Andreeva, 2012).

Assessment of skin fold (ASF): The applied methodology resulted in reduction of resistance and pain through the management of ASF in all three study areas. Despite the lack of statistically significant results, the observed skin folds showed a small to medium reduction results. The most outstanding changes were seen through management of the ASF in the medial part of the patella, followed by the navel-level area and finally by the trochanter major area.

Pain: the monitoring of the dynamics of pain symptoms indicates that the presence of severe pain before menstruation reveals a tendency to decrease significantly within the first month after initiation of treatment.

The results of the pain test before menstruation are presented in Table 1.

Table 1. Comparative analysis of the percent correlation to the experienced premenstrual pain

\begin{tabular}{|c|c|c|c|c|}
\hline \multicolumn{2}{|c|}{ Pain assessment } & Initial & Final & After 1 month \\
\hline No pain 0 & $\begin{array}{c}\text { patients } \\
\%\end{array}$ & $\begin{array}{c}0 \\
, 0 \% \\
\end{array}$ & $\begin{array}{c}2 \\
5,0 \% \\
\end{array}$ & $\begin{array}{c}10 \\
25,0 \%\end{array}$ \\
\hline Weak, dull pain 1 & $\%$ & $\begin{array}{c}1 \\
2,5 \% \\
\end{array}$ & $\begin{array}{c}16 \\
40,0 \% \\
\end{array}$ & $\begin{array}{c}10 \\
25,0 \% \\
\end{array}$ \\
\hline $\begin{array}{l}\text { Moderate pain } \\
\text { does not interfere } \\
\text { with normal activity } 2\end{array}$ & $\%$ & $\begin{array}{c}12 \\
30,0 \%\end{array}$ & $\begin{array}{c}2 \\
5,0 \%\end{array}$ & $\begin{array}{c}0 \\
0,0 \%\end{array}$ \\
\hline $\begin{array}{l}\text { Severe pain, } \\
\text { hindering normal activity } 3\end{array}$ & $\%$ & $\begin{array}{c}7 \\
17,5 \%\end{array}$ & $\begin{array}{c}0 \\
0,0 \%\end{array}$ & $\begin{array}{c}0 \\
0,0 \%\end{array}$ \\
\hline
\end{tabular}

Before the onset of the treatment, the relative share of patients with very severe pain that prevented normal activity was $17.5 \%$ (7), with sense of moderate pain - $30.0 \%$ (12), and with low sense of pain - $2.5 \%$ (1). In the final study, patients who experienced severe pain which is inhibiting the normal activity were not identified. With an average degree of pain and following the application of the spot massage method, the patients drastically decreased to - $5.0 \%$ (2). The decrease of the patients with moderate pain, respectively, resulted in an increase of the patients with improvement and with weak sense of pain - 40.0\% (16). Patients who no longer experience pain are now up to $5.0 \%$ (2).

The strongest post-therapeutic effect is seen 1 month after the end of treatment, which is expressed in the good control of the sick feeling. There were no more patients experiencing severe pain. Even more, there are no patients experiencing moderate pain. Full control of the severe and moderate pain con- sequently increased and equalized the number of patients with low sense of pain and those who had no pain at all $-25.0 \%(10)$.

\section{DISCUSSION}

Several more important summaries and trends can be derived from the results obtained.

Pain is a complex and individual feeling that is difficult to evaluate, explore, manage and heal.

The results in the dynamics of the change of those three measured laps are probably a result of the different aspects of the body structure and composition as well as the individual peculiarities and receptivity to the applied methodology. Perhaps the reduction in the measured tours is due to regulation of metabolism, and according to MC theory the circulation of the liquid substance sue in the energy channels and body parts, as a result of applying the point massage, improves significant- 
ly. Ensuring easy passage through the channels is a guarantee of the smooth flow of the fluid substance and the liquid substance of the energy, which in turn regulates the function of the internal organs (dzang'fou-organs) (Goranova, 1994; Goranova, 2001) and respectively affects the measured laps.

The applied methodology resulted in a decrease to the resistance of the skin folding in all three areas. In the period from the control to the final measurement, most pronounced are the changes as it goes to keeping the skin fold of the medial part of the patella, followed by the zone at the navel level and finally the zone of the trochanter major. At the navel level and at the trochanter major, a decrease of the average arithmetic values was reported 1 month after discontinuation of the treatment in result of the skin folding.

Only when conducting a skin fold in the medial part of the patella, changes that have occurred till the end of the treatment are maintained. However, those differences remain unchanged up to 1 month after the completion of the procedures. Since this area is a reflexogenic area of the uterus, it is assume that due to congestive events in the uterus, for a better treatment results, a prolonged treatment is required.

As a consequence of the applied methodology, the resistance of the tissues and the stagnant phenomena are overcome, improving the drainage and the micro-circulation in the three reflex zones, as a result of which the "orange skin" is smoothed out.

During the MC, fluid retention is often observed, which compresses the lymphatic channels and the venules. This disturbs the micro-circulation and drainage of the skin, prevents the drainage of the tissue fluid and of the lymph, therefore leading to stasis and local edema. After applying the point massage method, the overall appearance of all the girls improved, the skin is now smooth, the body circumference is reduced at all of the monitored levels, and a weight reduction is achieved (although this was not the subject of our study). Probably the results are due to the improved micro-circulation and trophy of the treated areas, the increased supply of oxygen to the peripheral tissues and the stimulated metabolism. All improvements have had a positive effect on the general condition of women and has significantly reduced premenstrual pain.

\section{CONCLUSION}

The results of this study clearly show that point massage treatment has a good therapeutic effect and can be used effectively in order to reduce premenstrual symptoms. Application of point massage has resulted with high efficacy in controlling pain before menstruation, strong reducing effect of tracked circumferences, as well as a reduction in skin resistance.

The overall analysis of the results to the conducted study gives us the reason to conclude that the applied point method massage has a strong therapeutic effect in the control of all of the monitored indicators. The obtained good results from the application of Chinese point massage, suggest that this method can be used in kinesitherapeutic practice as a means of active treatment. Women with PMS compared to those without experiencing it, need specific care and comprehensive prevention measures.

For optimal results, it is desirable that regular moderate physical activity and balanced nutrition are combined (Ilinova, 2014; Vanljan, Dimitrova, 2017). The aspects which depend on the PMS are various, therefore it is consider that a detailed study, development and the use of new physical means will contribute to the optimal treatment.

\section{REFERENCES}

Andonova, T. (2014). Methodology for reducing stress and anxiety. Sports and Science, 4, Sofia, p.103-111.

Ford O, Lethaby A, Roberts H, Mol BWJ. (2009). Progesterone for premenstrual syndrome. In: The Cochrane Library, Issue 2. Chichester, UK: John Wiley \& Sons, Ltd. Search date 2008.

Goranova, Z. (1994). ANMO - Chinese healing massage. Sofia.

Goranova, Z. (2001). Chinese Traditional Methods of Treatment. Vol. 1, Collection of articles. Sofia. p.70-75.

Goranova, Z. (2006). Dysmenorrhea - mastering through physical methods. In: Kinesitherapy and Rehabilitation, Sofia, vol. 1-2, pp.30-38.

Goranova, Z. (2006). Massage with honey - a method of prophylaxis and treatment. In: Kinesitherapy and Rehabilitation, Sofia, vol. 1-2, pp. 53-56.

Goranova, Z. (2018). Protection of pain syndrome in gynecology - Dysmenorrhea. Acupuncture treatment. C: Chinese methods of treatment. Vol. 3, Collection of articles, Sofia, NSA PRESS 2018, pp. 132-153. 
Gotova, G., Filipova, M., Popova, D. (2015). Investigation of the therapeutic effect of auriculotherapy in the acute period of pain syndrome in lumbar spine in athletes. Physiotherapy, № 1-2, 2015, pp. 30-32.

Gotova, Z. (2015). Application manipulative Terrier massage in the lumbar region in patients with chronic faceted symptoms. Activities in Physical Education and Sport, 5 (2), pp. 180-182.

Gotova, Z. (2015). Parallelogram of cervicolumbar syndrome and application of Chinese point massage for treatment and prevention. Research in Kinesiology, 43 (2) pp. 166-169.

Haywood, A., Slade, P. (2007). Psychosocial associates of PMS and the moderating role of social support in a community sample. 62: 9-13.

Ilinova, B. (2014). Women and Sports. Avangard Prima Publishing House; Sofia: 42-50; 86-87.

Kedikova-Andreeva, S. (2012). Menstrual abnormalities in overweight girls in puberty and adolescence. Sofia.

Kraev, T. (2016). Healing Massage and Achievement of

\section{Relaxation. Sofia.}

Lee, M. Chang S. Kang D. (2004). Effects of SP6 acupressure on labor pain and length of delivery time in women during labor. J Altern Complement Med. 10:959-965.

Pinar, G., Colak, M. (2011). PMS in Turkish college students and its effect on life quality. 2-21-27.

Topuzov, I. (2000). Cellulite - characteristic and complex treatment. Sofia.

Vanljan G., Dimitrova D. (2017). Study on the factors for menstrual dysfunction in female athletes. International Scientific Congress of Applied Sports Sciences: proceedings book / NSA "Vasil Levski ", Sofia. 1, pp. 42-49.

\section{Corresponding author:} Tatyana Tomova, PhD

Assistant, National Sport Academy "Vassil Levski" Department of Sport medicine Sofia, Bulgaria

E-mail: tomova.tatyana@abv.bg 